\title{
Experimental Fracture Model versus Osteotomy Model in Metacarpal Bone Plate Fixation
}

\author{
S. Ochman, ${ }^{1}$ T. Vordemvenne, ${ }^{1}$ J. Paletta, ${ }^{2}$ M. J. Raschke, ${ }^{1}$ R. H. Meffert, ${ }^{3}$ and S. Doht ${ }^{3}$ \\ ${ }^{1}$ Department of Trauma, Hand and Reconstructive Surgery, University of Münster, \\ Albert Schweitzer Campus 1, Building W1, 48149 Münster, Germany \\ ${ }^{2}$ Department of Orthopaedic Surgery, Philipps-University Marburg, \\ 35043 Marburg, Germany \\ ${ }^{3}$ Department of Trauma, Hand, Plastic and Reconstructive Surgery, \\ University of Würzburg, 97070 Würzburg, Germany
}

Received 26 July 2011; Accepted 23 September 2011

Academic Editor: James Huntley

Introduction. Osteotomy or fracture models can be used to evaluate mechanical properties of fixation techniques of the hand skeleton in vitro. Although many studies make use of osteotomy models, fracture models simulate the clinical situation more realistically. This study investigates monocortical and bicortical plate fixation on metacarpal bones considering both aforementioned models to decide which method is best suited to test fixation techniques. Methods. Porcine metacarpal bones $(n=40)$ were randomized into 4 groups. In groups I and II bones were fractured with a modified 3-point bending test. The intact bones represented a further control group to which the other groups after fixation were compared. In groups III and IV a standard osteotomy was carried out. Bones were fixated with plates monocortically (group I, III) and bicortically (group II, IV) and tested for failure. Results. Bones fractured at a mean maximum load of $482.8 \mathrm{~N} \pm 104.8 \mathrm{~N}$ with a relative standard deviation (RSD) of $21.7 \%$, mean stiffness was $122.3 \pm 35 \mathrm{~N} / \mathrm{mm}$. In the fracture model, there was a significant difference $(P=0.01)$ for maximum load of monocortically and bicortically fixed bones in contrast to the osteotomy model $(P=0.9)$. Discussion. In the fracture model, because one can use the same bone for both measurements in the intact state and the bone-plate construct states, the impact of inter-individual differences is reduced. In contrast to the osteotomy model there are differences between monocortical and bicortical fixations in the fracture model. Thus simulation of the in vivo situation is better and seems to be suitable for the evaluation of mechanical properties of fixation techniques on metacarpals.

KEYWORDS: biomechanics, fracture model, metacarpal, osteotomy, plate fixation 


\section{INTRODUCTION}

A technological progression with an increase in the number and design of implants "like angular stable plates" to assure fracture healing and functional recovery has succeeded in hand surgery [1-4]. Variations in fracture models and biomechanical testing methods make it difficult to compare results among different studies. Most investigators used an osteotomy model and a four- or three-point bending protocol. A homogeneous fracture type is beneficial for the osteotomy model. However, the SD of these models is very variable [5-7].

By contrast, a fracture model could simulate the clinical situation more realistically. The purpose of this study was to create a consistent metacarpal fracture model in vitro using porcine metacarpals. This study investigates monocortical and bicortical plate fixation comparing the osteotomy and fracture models to decide which method is best to test fixation techniques.

\section{MATERIAL AND METHODS}

Fresh second metacarpal bones from 28-week-old domestic pigs were used $(n=40)$. Specimens were dissected from soft tissue; their dimensions were measured (average anterior-posterior diameter $5.26 \mathrm{~mm}$ ) and stored at $-20^{\circ} \mathrm{C}$ to preserve their mechanical properties close to those of fresh bones [8]. Specimens were embedded in a fixation device ( $14 \mathrm{~mm}$ of the proximal bone) using Palacos (Kulzer GmbH, Wehrheim, Germany). The free length was $25 \mathrm{~mm}$ to the center of the metacarpal head. All specimens were allowed to equilibrate to room temperature and were kept moist with saline irrigation during preparation, surgical procedure, and biomechanical testing.

Specimens were randomly divided into 4 groups. In two groups, a midshaft fracture was generated (groups I and II) using a modified 3-point bending method (Zwick/Roell, Z005/TN2A, Ulm, Germany) (Figure 1). Load to failure was applied with a constant speed of $100 \mathrm{~mm} / \mathrm{min}$. Data were collected to ascertain the maximum load and the yield load and also to calculate bone stiffness. In the other two groups, a midshaft transverse osteotomy, $15 \mathrm{~mm}$ proximal to the embedded bone side, was carried out (groups III and IV).

The fractures were dorsally reduced and fixed using a titanium miniplate (thickness $1.0 \mathrm{~mm}, 4$ holes and $2.3 \mathrm{~mm}$ screws) and the midpoint lined up with fracture or osteotomy side (Leibinger-Stryker, Freiburg, Germany). Fixation was monocortical (group I, group III) or bicortical (group II, group IV). All fixations were carried out standardised by one surgeon. Two nonlocking screws were placed on each side of the fracture right-angled to the plate.

Biomechanical testing was performed in the same way the fracture was caused. In the osteotomy groups, a lateral fixation device was used to reduce the rotation of the bones. Load-deflection curves were continuously recorded and the ultimate failure [N] was measured. Stiffness [6] was determined from the linear region of the load-displacement curves. Data was collected using testXpert V10.11 (Zwick/Roell).

Mean value and SD were calculated for each test group and for intact bones using Microsoft Excel (Microsoft, Bellevue, Wash, USA). Failure points and stiffness were compared with nonparametric KruskalWallis test using SPSS 15.0. Statistical significance was set at a value of $P<0.05$.

\section{RESULTS}

Intact bones showed a highly reproducible pattern of shaft fracture similar to AO A2 with a relative standard deviation of $21.7 \%$ (Figure 2, Table 1).

Between maximum load of intact bone and after bicortical $(R 2=0.817)$ and monocortical $(R 2=$ 0.665 ) fixation, there was a linear correlation. The monocortical-fixed bones reached mean $53.7 \%$ (SD $10.7 \%)$ of the maximum load of native bones $(P=0.049)$. With regard to bicortical fixation the construct reached mean $73.1 \%$ (SD 14\%) $(P=0.015)$. Regarding stiffness and yield load bicortical-fixed bones 


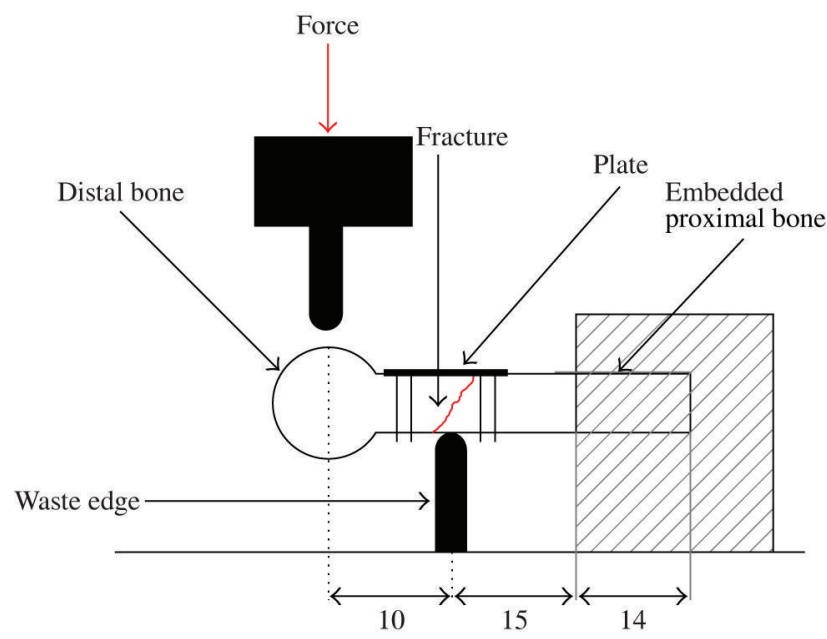

FIGURE 1: Test set up: embedded specimens were placed on a waste edge in a certain position (distance: fixation device-waste edge: $15 \mathrm{~mm}$ ). Load application was apical distal (distance: waste edge-point of force transmission: $10 \mathrm{~mm}$ ) with a constant speed of $100 \mathrm{~mm} / \mathrm{min}$ until failure was noted.

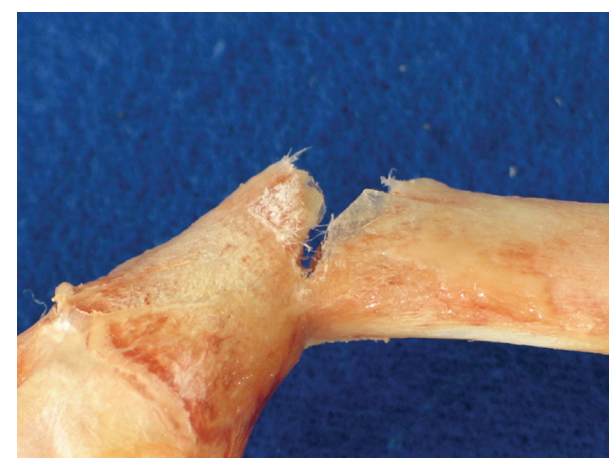

FIGURE 2: Metacarpal fracture line passes from the dorsal cortex to the volar cortex short oblique or transverse (AO A2 fracture type).

reached higher values compared to the native bones (stiffness mean 49.9\% SD 25.1\%, $P=0.893$; yield load mean $82.8 \%$ SD $20.6 \%, P=0.063$ ) than the monocortical-fixed bones (stiffness mean $40.9 \%$, SD $14.5 \%, P=0.910$, yield load mean 58.8\%, SD 18.9\%, $P=0.114$ ) (Figure 3, Table 1).

The comparison of group III (osteotomy, monocortical) and group IV (osteotomy, bicortical) shows no significant differences in terms of maximum load $(338.4 \mathrm{~N} \pm 79.2 \mathrm{~N}$ versus $343.9 \mathrm{~N} \pm 88.5 \mathrm{~N}, P=$ $0.910)$, yield load $(219.3 \mathrm{~N} \pm 96 \mathrm{~N}$ versus $236.4 \pm 69.4 \mathrm{~N}, P=0.579)$, and stiffness $(49.5 \mathrm{~N} / \mathrm{mm} \pm$ $23.5 \mathrm{~N} / \mathrm{mm}$ versus $58.9 \mathrm{~N} / \mathrm{mm} \pm 16.3 \mathrm{~N} / \mathrm{mm}, P=0.165$ ) (Table 1 ).

In the fracture model, however, there was a significant difference in maximum load $(P=0.01)$ between monocortical (mean 250 N SD 56.1 N) and bicortical (mean 359.4 N SD 90.4 N) fixation (Table 1). Yield load $(171.5 \mathrm{~N} \pm 54.7$ versus $253.8 \mathrm{~N} \pm 77.1 \mathrm{~N}, P=0.019)$ and stiffness $(46.5 \mathrm{~N} / \mathrm{mm} \pm 12.6 \mathrm{~N} / \mathrm{mm}$ versus $56.1 \mathrm{~N} / \mathrm{mm} \pm 21.1 \mathrm{~N} / \mathrm{mm}, P=0.393$ ) of these groups demonstrated no significant differences.

Failure was located at the osteotomy with displacement of the apex. In the fracture model there was a bending of the plate with displacement at the fracture and a failure at the screw-bone interface. 


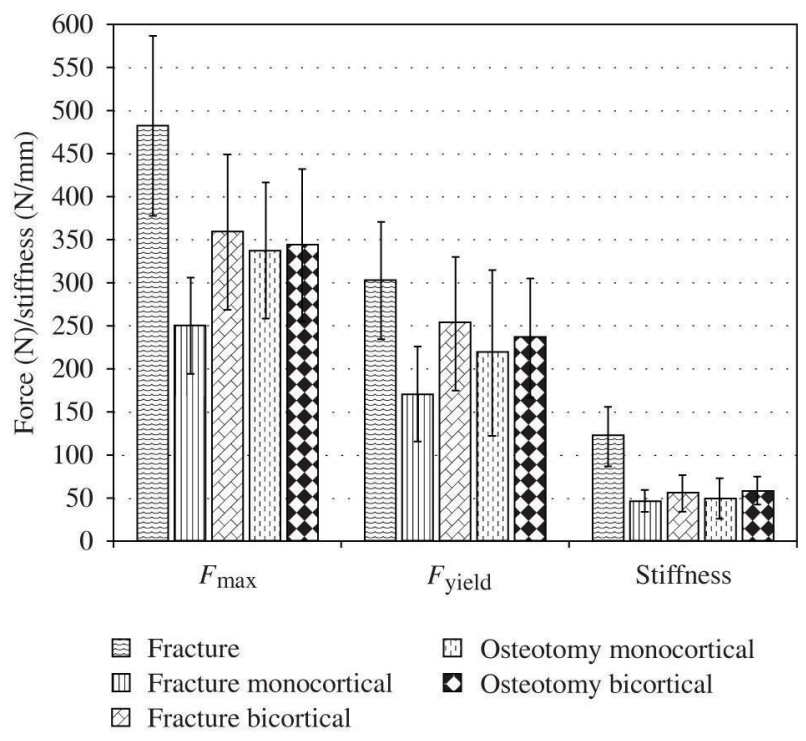

FIGURE 3: Maximum load $(\mathrm{N})$, yield load $(\mathrm{N})$, and stiffness $(\mathrm{N} / \mathrm{mm})$ of native bones, osteotomy and fracture model.

TABLE 1: Maximum load $\left(F_{\max }\right)$, Yield-load ( $\left.F_{\text {yield }}\right)$, and stiffness of native bones, monocortical- and bicortical-fixed bones in the osteotomy and fracture model.

\begin{tabular}{|c|c|c|c|c|c|c|c|c|}
\hline & & & & Mean & Range & SD & RSD [\%] & $\%$ \\
\hline & & & $F_{\max }[\mathrm{N}]$ & 482.8 & $360.6-779.2$ & 104.8 & 21.7 & 100 \\
\hline Native $b$ & one fracturi & $g(n=20)$ & $F_{\text {yield }}[\mathrm{N}]$ & 303.1 & $200-500$ & 68.1 & 22.5 & 100 \\
\hline & & & $\begin{array}{c}\text { Stiffness } \\
{[\mathrm{N} / \mathrm{mm}]} \\
\end{array}$ & 122.3 & $67.4-207.2$ & 35.0 & 28.6 & 100 \\
\hline & & & $F_{\max }[\mathrm{N}]$ & 250.0 & $160.1-320.4$ & 56.1 & 22.4 & 51.8 \\
\hline & & $(n=10)$ & $F_{\text {yield }}[\mathrm{N}]$ & 171.5 & $90-255.7$ & 54.7 & 31.9 & 56.6 \\
\hline & $\begin{array}{l}\text { cortical } \\
\text { fixed }\end{array}$ & & $\begin{array}{l}\text { Stiffness } \\
{[\mathrm{N} / \mathrm{mm}]}\end{array}$ & 46.5 & $29.1-67.8$ & 12.6 & 27.0 & 38.0 \\
\hline & & & $F_{\max }[\mathrm{N}]$ & 338.4 & $209.7-443.3$ & 79.2 & 23.4 & 70.1 \\
\hline Bone plate & & $(n=10)$ & $F_{\text {yield }}[\mathrm{N}]$ & 219.3 & $117.3-335.3$ & 96.0 & 43.8 & 72.4 \\
\hline & & & $\begin{array}{l}\text { Stiffness } \\
{[\mathrm{N} / \mathrm{mm}]}\end{array}$ & 49.5 & $28.5-101.7$ & 23.5 & 47.4 & 40.5 \\
\hline & & & $F_{\max }[\mathrm{N}]$ & 359.4 & $210.6-491.3$ & 90.4 & 25.1 & 74.4 \\
\hline & & $(n=10)$ & $F_{\text {yield }}[\mathrm{N}]$ & 253.8 & $150-389.1$ & 77.1 & 30.4 & 83.7 \\
\hline & $\begin{array}{l}\text { Bicortical } \\
\text { fixed }\end{array}$ & & $\begin{array}{l}\text { Stiffness } \\
{[\mathrm{N} / \mathrm{mm}]}\end{array}$ & 56.1 & $27.5-98.8$ & 21.1 & 37.6 & 45.9 \\
\hline & & & $F_{\max }[\mathrm{N}]$ & 343.9 & $222.7-456.3$ & 88.5 & 25.7 & 71.2 \\
\hline & & $(n=10)$ & $F_{\text {yield }}[\mathrm{N}]$ & 236.4 & $150.2-346.2$ & 69.4 & 29.4 & 78.0 \\
\hline & & & $\begin{array}{l}\text { Stiffness } \\
{[\mathrm{N} / \mathrm{mm}]}\end{array}$ & 58.9 & $37.6-85.8$ & 16.3 & 27.6 & 48.2 \\
\hline
\end{tabular}




\section{DISCUSSION}

The objective of operative treatment of metacarpal fractures should be anatomical reconstruction and stable fixation for early functional therapy. There are a variety of fracture types including complex fractures with articular involvement to which it is difficult to achieve adequate fragment fixation.

Dorsal plating provides superior biomechanical fixation compared to other techniques. [9-12] The predominant force acting is apex dorsal loading and bending in volar direction due to the action of flexor tendons. A dorsal plate position with at least two screws on each side of the fracture provides the greatest rigidity to an apex applied load $[9,10]$. Using a standard osteotomy procedure we could not demonstrate any differences between mono- and bicortical fixations. These results are similar to results from Dona et al. 2004, testing dorsal mono- and bicortical plating in a cadaveric metacarpal osteotomy [6].

The disadvantage using human specimens is that they are not homogenous samples. By contrast, animal specimens have a relatively uniform mechanical strength with similar shape, size, and bone mineral density. Like other investigators we used porcine second metacarpal bones [13, 14]. The diaphyseal region of the nonweight bearing second metacarpal porcine bone is comparable to human metacarpal and proximal phalanx bones $[11,13]$. Metacarpal bones of 28-week-old domestic pigs were used which had just reached skeletal maturity. Therefore, the fracture model correlated to fractures in young adults and did not simulate osteoporotic fractures in elderly patients.

Having created a fracture protocol we could demonstrate a significant higher stability of bicortical plate fixation as compared with monocortical plate fixation. This reconfirms our experience from clinical observations. The fracture model shows a relative standard deviation (RSD) for the maximum load of $21.7 \%$ for fracturing the native bones. Relative standard deviation (RSD) for maximum load after bicortical and monocortical fixation in the fracture model was $22.4 \%$ and $25.1 \%$, for all bones in the fracture model $24.41 \%$. Comparing to the osteotomy model RSD for maximum load $\left(F_{\max }\right)$ in the fracture model was even lower $(24.41 \%$ versus $35 \%$ ). The RSD of osteotomy models in the literature is very variable with $30.75 \%$ in load to failure of porcine metacarpal bones [6]. The RSD of human metacarpal osteotomy model testing bioabsorbable plates was $27.6 \%$ [5] and on a sawbone osteotomy model 5.6\% [7].

Another benefit of the fracture protocol is the possibility to measure a maximum load of the bones to cause a fracture as well as to test the maximum load of the plate-bone construct on the same bone. This procedure considers the interindividual variations of the bones and it allows the determination of a "relative maximum load" for each specimen (= normalized values compared to native properties). Furthermore it could simulate the clinical situation in a better way than an osteotomy.

Influences of the soft tissue especially tendons were not considered in this model. The standard procedure of fracture stabilization with plates is a bicortical position of the screws including drilling through both dorsal and volar cortices, which places the flexor tendons and neurovascular bundles at risk. Moreover, the correct screw size is critical and the screw tip can interfere with the flexor tendons and cause tendon rupture $[15,16]$. Statistically there was no significant difference between mono- and bicortical fixation of cadaveric metacarpal bones using dorsally conventional plating [6]. This is similar to our findings but it does not reflect our clinical observations. Using the fracture model, there was a significant difference between mono- and bicortical plate fixation comparable to clinical investigations. Open reduction in the fracture model shows an interlocking of the cortical bone at the fracture site. This prevents rotational dislocation which could be a possible mechanism of failure in the osteotomy setting.

In several biomechanical studies, metallic and bioabsorbable fixation devices of metacarpal and phalangeal osteotomies have been investigated [13, 14, 17]. Metallic plate fixation with an additional interfragmentary screw is biomechanically superior to other fixation methods [9]. In our study we only tested plate fixation without interfragmentary screws.

The type of failure in our study was deformation or cortex breaking at the fracture site with cutting out of the screws at the cortex. Bioabsorbable plates failed through plate or bone cortex breaking, showing the same modulus of elasticity of the plate and the cortex [14]. 
The present paper has a number of limitations. We tested static unidirectional load only. This testing method provides valuable measurements but does not reflect the clinical situation of fixation devices in hand surgery. A cyclical testing protocol is currently under review. Because of the predominantly dorsal apex loading we tested this load direction only. The influence of torsion or sudden loading cannot be estimated. In vivo loading will be more complex and certain clinical settings may include a torsional component.

Using our model, fractures are reproducible with a low variation. Because one can use the same bone for both measurements, in the intact state and the bone-plate construct state, the impact of interindividual differences is reduced. In contrast to the osteotomy model it shows differences between monocortical and bicortical fixations comparable to clinical investigations. It simulates the in vivo situation realistically and seems to be suitable for the evaluation of mechanical properties of fixation techniques on metacarpals.

\section{AUTHOR CONTRIBUTION}

First and second author contributed in equal parts.

\section{ACKNOWLEDGMENTS}

The authors would like to thank Steffen Schanz, M.S. from the Biomechanical Laboratory, Department of Trauma, Hand and Reconstructive Surgery, University of Muenster for the excellent support regarding the concept of the study and the acquisition of data. Furthermore, they would like to thank Wolfgang Walch, Transmedical, Ireland for the comprehensive review of the paper. Financial support for fixation devices and plates has been received from Leibinger/Stryker, Freiburg.

\section{REFERENCES}

[1] A. E. Freeland, W. B. Geissler, and A. P. Weiss, "Surgical treatment of common displaced and unstable fractures of the hand," Instructional Course Lectures, vol. 51, pp. 185-201, 2002.

[2] A. E. Freeland and S. G. Lindley, "Malunions of the finger metacarpals and phalanges," Hand Clinics, vol. 22, no. 3, pp. 341-355, 2006.

[3] S. H. Kozin, J. J. Thoder, and G. Lieberman, "Operative treatment of metacarpal and phalangeal shaft fractures," The Journal of the American Academy of Orthopaedic Surgeons, vol. 8, no. 2, pp. 111-121, 2000.

[4] P. J. Stern, "Management of fractures of the hand over the last 25 years," Journal of Hand Surgery, vol. 25, no. 5, pp. 817-823, 2000.

[5] K. J. Bozic, L. E. Perez, D. R. Wilson, P. G. Fitzgibbons, and J. B. Jupiter, "Mechanical testing of bioresorbable implants for use in metacarpal fracture fixation," Journal of Hand Surgery, vol. 26, no. 4, pp. 755-761, 2001.

[6] E. Dona, R. M. Gillies, M. P. Gianoutsos, and W. R. Walsh, "Plating of metacarpal fractures: unicortical or bicortical screws?" Journal of Hand Surgery, vol. 29, no. 3, pp. 218-221, 2004.

[7] R. C. Sohn, K. H. Jahng, S. B. Curtiss, and R. M. Szabo, "Comparison of metacarpal plating methods," Journal of Hand Surgery, vol. 33, no. 3, pp. 316-321, 2008.

[8] C. H. Turner and D. B. Burr, "Basic biomechanical measurements of bone: a tutorial," Bone, vol. 14, no. 4, pp. 595-608, 1993.

[9] D. Black, R. J. Mann, R. Constine, and A. U. Daniels, "Comparison of internal fixation techniques in metacarpal fractures," Journal of Hand Surgery, vol. 10, no. 4, pp. 466-472, 1985.

[10] R. J. Mann, D. Black, R. Constine, and A. U. Daniels, "A quantitative comparison of metacarpal fracture stability with five different methods of internal fixation," Journal of Hand Surgery, vol. 10, no. 6, part 2, pp. 1024-1028, 1985.

[11] J. B. Massengill, H. Alexander, J. R. Parson, and M. J. Schecter, "Mechanical analysis of Kirschner wire fixation in a phalangeal model," Journal of Hand Surgery, vol. 4, no. 4, pp. 351-356, 1979. 
[12] J. A. Nunley and P. Kloen, "Biomechanical and functional testing of plate fixation devices for proximal phalangeal fractures," Journal of Hand Surgery, vol. 16, no. 6, pp. 991-998, 1991.

[13] J. B. Massengill, H. Alexander, N. Langrana, and A. Mylod, "A phalangeal fracture model—quantitative analysis of rigidity and failure," Journal of Hand Surgery, vol. 7, no. 3, pp. 264-270, 1982.

[14] E. Waris, N. Ashammakhi, H. Happonen et al., "Bioabsorbable miniplating versus metallic fixation for metacarpal fractures," Clinical Orthopaedics and Related Research, no. 410, pp. 310-319, 2003.

[15] R. A. Fambrough and D. P. Green, "Tendon rupture as a complication of screw fixation in fractures in the hand. A case report," Journal of Bone and Joint Surgery-Series A, vol. 61, no. 5, pp. 781-782, 1979.

[16] S. M. Page and P. J. Stern, "Complications and range of motion following plate fixation of metacarpal and phalangeal fractures," Journal of Hand Surgery, vol. 23, no. 5, pp. 827-832, 1998.

[17] K. K. Firoozbakhsh, M. S. Moneim, T. Howey, E. Castaneda, and M. A. Pirela-Cruz, "Comparative fatigue strengths and stabilities of metacarpal internal fixation techniques," Journal of Hand Surgery, vol. 18, no. 6, pp. 1059-1068, 1993.

\section{This article should be cited as follows:}

S. Ochman, T. Vordemvenne, J. Paletta, M. J. Raschke, R. H. Meffert, and S. Doht, "Experimental Fracture Model versus Osteotomy Model in Metacarpal Bone Plate Fixation," TheScientificWorldJOURNAL, vol. 11, pp. 1692-1698, 2011. 


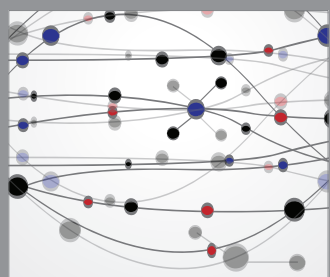

The Scientific World Journal
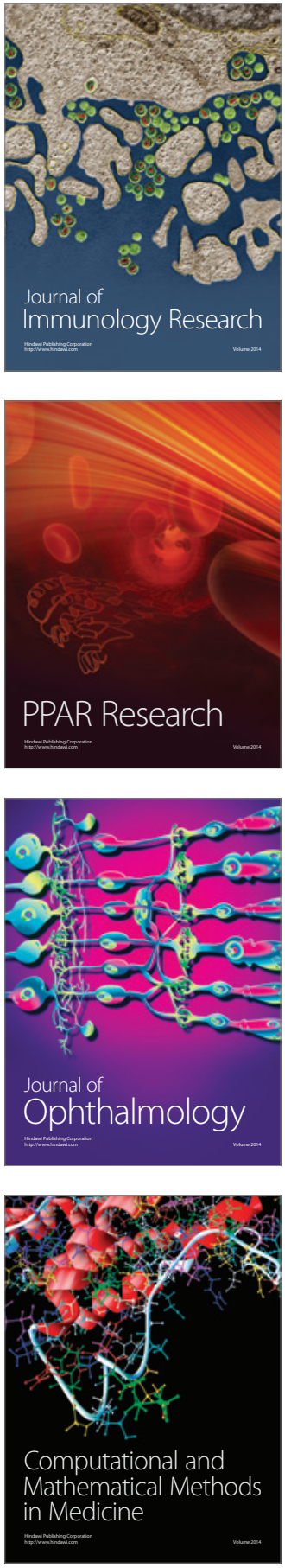

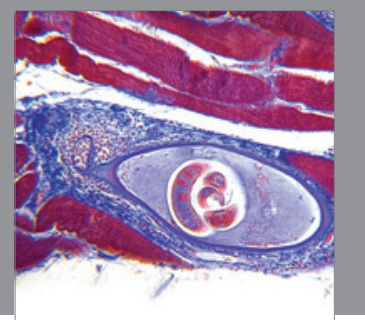

Gastroenterology

Research and Practice
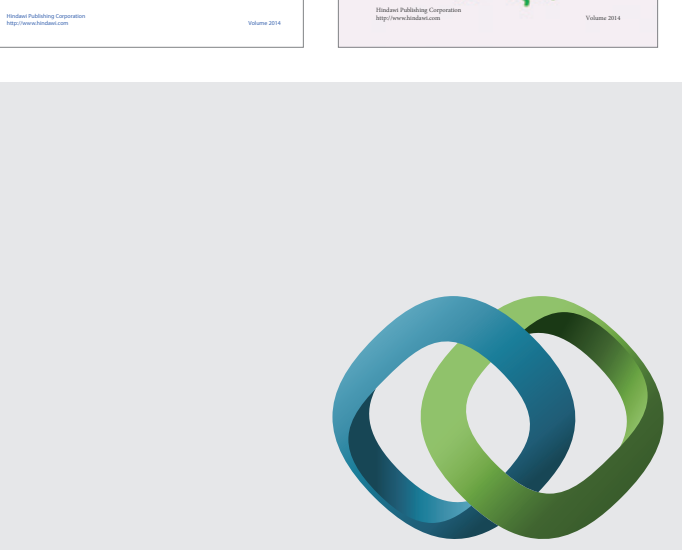

\section{Hindawi}

Submit your manuscripts at

http://www.hindawi.com
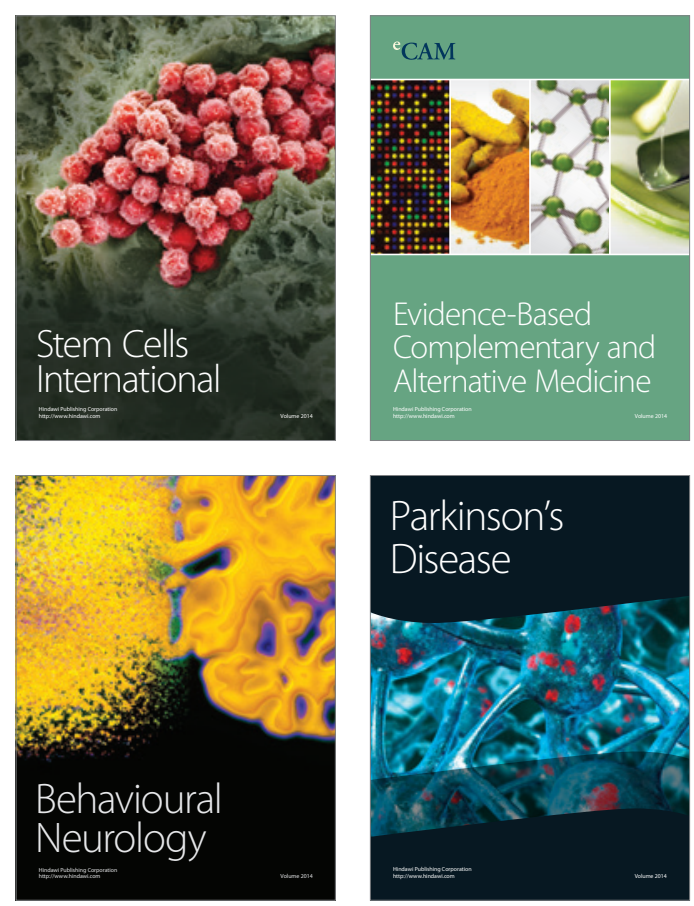

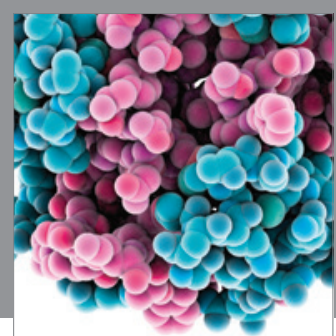

Journal of
Diabetes Research

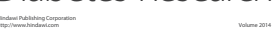

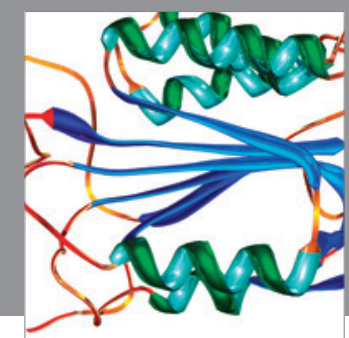

Disease Markers
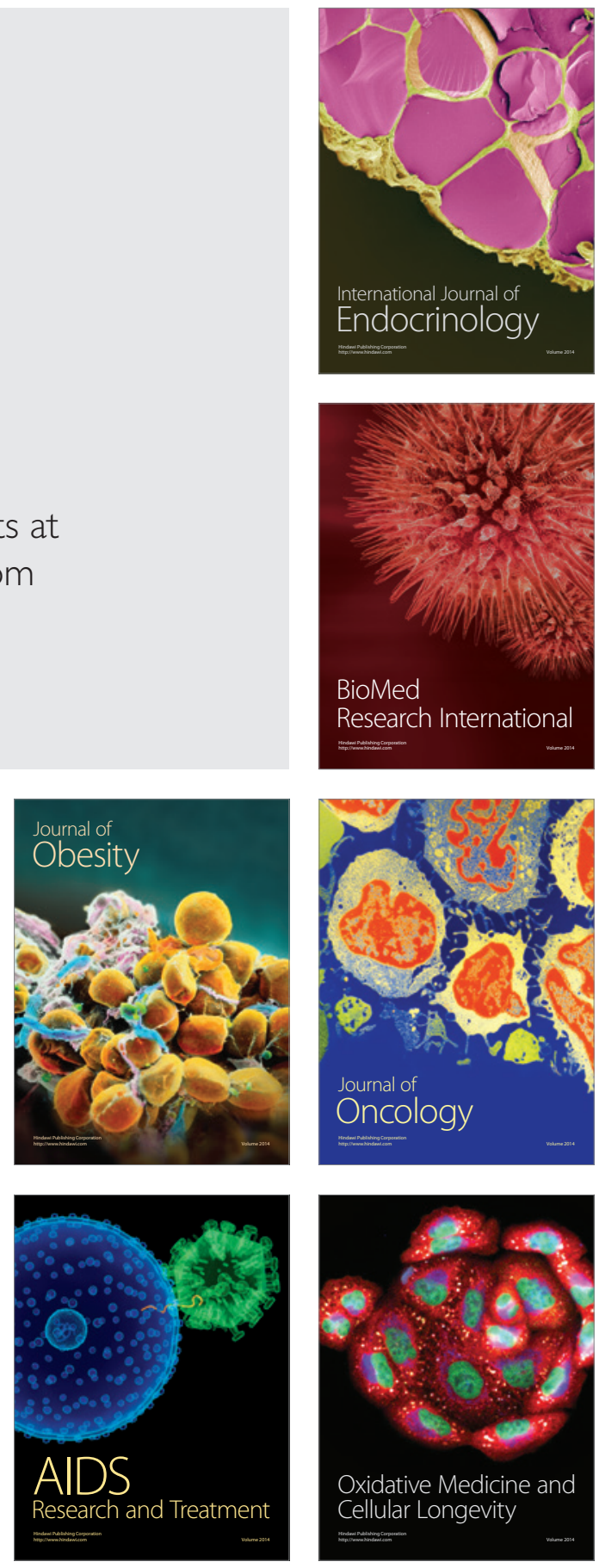